\title{
OUTBREAK OF ROSS RIVER VIRUS DISEASE IN THE SOUTH WEST DISTRICTS OF NSW, SUMMER 1993
}

Louise McDonnell

Public Health Officer, Western Sector Public Health Unit

Tony Kolbe, Acting Director,

Terry Carvan, Environmental Health Officer,

Kim Gilchrist, Public Health Officer,

South West Centre for Public Health

A large outbreak of epidemic polyarthritis (EPA) caused by the arbovirus Ross River virus (RRV) occurred in the south west of NSW during the summer of 1993. A total of 312 cases was reported by laboratories in contrast to 33 cases in 1991 and 29 cases in 1992. Cases occurred from January to June, with 64 per cent occurring in January and February. The outbreak followed heavy rains between October and December 1992. This report describes the outbreak of RRV disease which was the largest in the south west since the Statewide RRV outbreak in 1984 (Figure 5).

The major inland vector of RRV in NSW is the mosquito Culex annulirostris and the major coastal vector is Aedes vigilax. The clinical symptoms of RRV infection vary but commonly include rash, lethargy and myalgia followed by arthralgia, joint stiffness and joint swelling. A follow-up study of RRV patients in a Western Australian outbreak in 1988-89 showed that only 27 per cent of cases had fully recovered within six months. In addition, more than 33 per cent of cases had at least one month off work'. The economic consequences of RRV are likely to be large as attack rates are highest in the working-age groups"2.

\section{METHODS}

Notifications were received from five main laboratories two in NSW and three in Victoria. We defined a case as a resident of the South West Districts whose paired sera showed a fourfold or greater rise in antibody titre, or whose single serum specimen demonstrated RRV-specific IgM antibody. This was in accordance with the NSW Health Department Infectious Disease Manual.

Residence was taken as a proxy for location of infection. Date of the first serological test was used as an estimate of the date of onset of symptoms. We calculated linear trend, attack rates and relative risks with Epi Info version $5^{3}$.

\section{RESULTS}

A total of 312 patients with RRV disease was notified from the South West Districts of NSW from January to June 1993, which is a tenfold increase in notifications for the same period in 1992 (Figure 6). Cases appeared in January, peaked in February and had returned to pre-epidemic levels by May when only two cases were reported.

Notifications were grouped into local government areas (LGAs) according to residence. The highest attack rates occurred in the west of the region. Wentworth had the highest attack rate $(770 / 100,000)$ followed by Deniliquin $(539 / 100,000)$ and Hay $(387 / 100,000)$ (Figure 7$)$.

The youngest patient was eight years of age and the oldest was 83. Age-specific attack rates are shown in Table 2 . The highest attack rates were in the 30-49 age groups (linear trend test $\left.x^{2}=20.68 \mathrm{p}<0.0001\right)$. Female cases exceeded males by 173 to 135 (1.3:1) but this was not statistically significant. An anomaly was the relative risk of disease in women aged 60-69 years compared with men of the same age $(R R=9.87, p<0.0003)$

\section{FIGURE 5}

ARBOVIRAL NOTIFICATIONS IN THE

SOUTH WEST DISTRICTS 1983-1993*

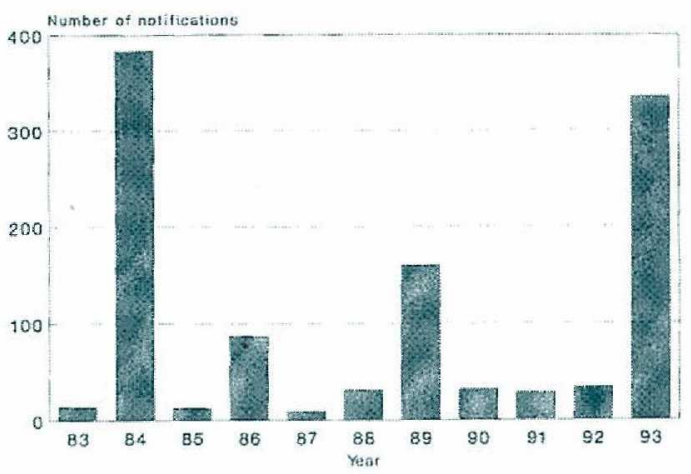

* The majority of notifications are RRV infections but those before 1989 are not serologically proven.

Source: IDSS, NSW Health Dept

\section{FIGURE 6}

NUMBER OF CASES OF RRV INFECTION BY MONTH OF ONSET, SOUTH WEST DISTRICTS JANUARY 1991-JUNE 1993

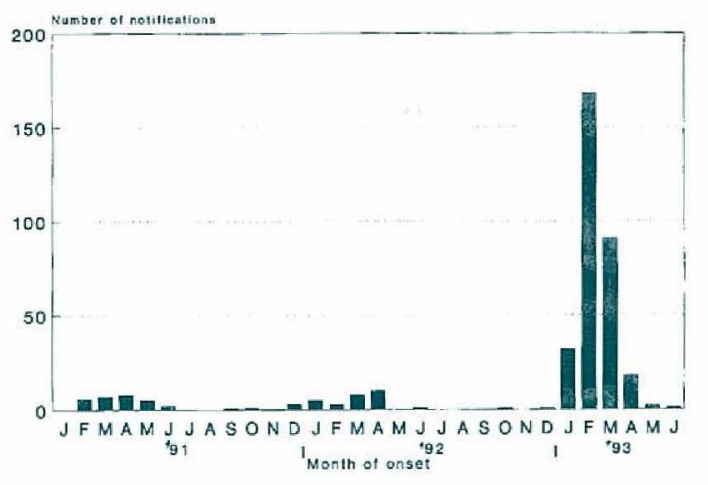

Source: IDSS, NSW Health Dept

\section{DISCUSSION}

This outbreak represents the largest number of RRV cases reported in the South West Districts since the Statewide RRV outbreak in NSW in 1984, which was preceded by a long drought, followed by excessive January rainfall. In the 1993 outbreak, heavy rains occurred from October to December 1992 and January rainfall was average. January temperatures in 1993 were below average and it is likely this delayed and reduced the size of the outbreak.

Residential address has limitations as a proxy for location of infection because cases may have been bitten elsewhere. Acknowledging this, there was a preponderance of cases in the centre and west of the region. These areas were flooded between October and December 1992, providing large areas of water for mosquito breeding. Irrigation used extensively in this area may have produced further breeding sites.

The reason for the large number of elderly female cases in this outbreak is unknown. Date of onset among the elderly 


\section{FIGURE 7}

MAP OF THE SOUTH WEST DISTRICTS SHOWING ATTACK RATES OF RRV INFECTION BY LOCAL GOVERNMENT AREA DURING THE 1993 OUTBREAK

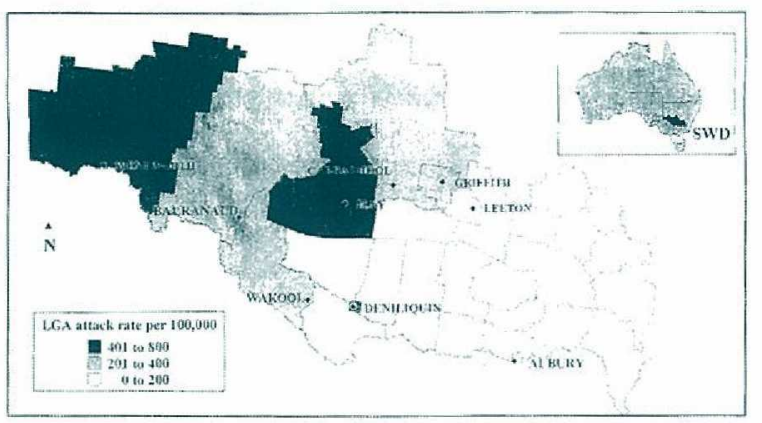

females varied widely and there was no suggestion of a common exposure. Children under nine years and adults over 70 experienced low attack rates. Little is known about the seroprevalence of arbovirus antibodies in children as most of the studies have been done in adults. It may be that children are infected with RRV but have mild illness which is unrecognised. The lower attack rates in the elderly may reflect a higher level of immunity as seroprevalence of RRV antibodies increases with age ${ }^{4}$.

The investigation of this outbreak has exposed several deficiencies in the NSW arbovirus notification system. In the South West Districts where RRV is endemic, many GPs make clinical diagnoses and do not order confirmatory serology. It is likely the laboratory-based notification system underestimates the true number of cases.

A recent article describes a case of $R R V$ infection as "confirmed" if seroconversion is demonstrated in paired sera and "presumptive" if RRV-specific IgM is present in a single specimen, or stable antibody levels are demonstrated in paired sera ${ }^{5}$. The NSW notification form does not provide sufficient details of the number of sera tested or results to allow cases to be classified as "confirmed" or "presumptive".

Arboviral antibodies are most commonly measured by an enzyme-linked immunosorbent assay (ELISA) method using either "laboratory-made" reagents or reagents from a commercially available kit. Assays using reagents from commercial kits are said to have 10-14 per cent false positive rate ${ }^{5}$, compared with a 3 per cent false positive rate reported by the Institute of Clinical Pathology and Medical Research, Westmead NSW, which uses a "laboratory-made" reagent based on neutralisation inhibition standards (personal communication, Ms Linda Huston). Knowledge of the laboratory and type of assay reagent used is important when interpreting RRV notification data. This information is not available on the NSW notification form.

Arboviral serology is complex and interpretation of results can be difficult. Many general practitioners make a diagnosis of RRV on a single positive IgM result. If IgG is also present, it may not be possible to determine whether the patient is seroconverting or has had a past infection. RRV IgM usually indicates recent infection but has been found in convalescent sera up to 12 months after initial infection ${ }^{6}$. GPs need education on the importance of paired sera and the limitations of a single IgM result.
TABLE 2

NUMBER OF CASES AND ATTACK RATE OF ROSS RIVER VIRUS INFECTION BY AGE GROUP, SOUTH WEST DISTRICTS, 1993

\begin{tabular}{|lrrr|}
\hline $\begin{array}{l}\text { Age group } \\
\text { in years }\end{array}$ & $\begin{array}{r}\text { Number } \\
\text { of cases }\end{array}$ & $\begin{array}{r}\text { Population } \\
\text { per age group }\end{array}$ & $\begin{array}{r}\text { Attack rate } \\
\text { per 100,000 }\end{array}$ \\
\hline $0-9$ & 5 & 42,674 & 11.7 \\
$10-19$ & 18 & 41,306 & 43.5 \\
$20-29$ & 57 & 36,903 & 154.3 \\
$30-39$ & 83 & 39,185 & 211.8 \\
$40-49$ & 67 & 31,662 & 211.6 \\
$50-59$ & 39 & 23,845 & 163.5 \\
$60-69$ & 22 & 21,589 & 101.9 \\
$70+$ & 9 & 19,018 & 47.3 \\
\hline Total & $* 312$ & 256,182 & 123.2 \\
\hline
\end{tabular}

*Includes 12 cases with unknown age.

If correlation of notifications with meteorological and mosquito data is to be attempted, details of location and date of infection must be known. This information is not recorded on the NSW notification forms. The Northern Territory Department of Health and Community Services uses a specific arboviral notification form, which documents date and place of infection, laboratory and assay used and serology results, including the number of sera tested. (Mr Peter Whelan, personal communication.) The form is completed by general practitioners at the time of diagnosis, or by the Health Department at the time of notification. Implementation of a similar form in NSW could greatly improve the system. Alternatively, RRV notifications could be managed only by Public Health Units servicing areas where RRV cases regularly occur. In this setting, a general practitioner sentinel system may be appropriate. If we are to continue with the current notification system, individual case follow-up to obtain these details is needed. This is timeconsuming and may be impractical during an outbreak.

Whatever system is chosen, our reasons for collating data on RRV infections should be clear. The information collected should enable the confirmation of a case and the likely place and time of infection to enable appropriate and timely control measures.

\section{ACKNOWLEDGMENTS}

The authors acknowledge Mr Peter Whelan and Ms Linda Huston for their comments and Dr Angela Merianos,

National Centre for Epidemiology and Population Health, for her advice and review of the manuscript.

1. Condon RJ, Rouse IL. Ross River Virus infection in the south-west of Western Australia, 1988-89: A follow-up study. Presented at the RACP, AFPM and AFRM Annual Scientific Meeting, Sydney, April 1993. 2. Hawkes RA, Boughton CR, Naim HM, Stallman ND. A major outbreak of epidemic polyarthritis in New South Wales during the outbreak of epidemic polyarthritis in New South Wale
summer of 1983-1984. Med J Aust 1985; 143:330-333. 3. Epi Info, Version 5. Dean AG, Dean JA, Burton AH, Dicker RC. US Department of Health and Human Services.

4. Boughton CR, Hawkes RA, Naim HM, Wild J, Chapman B. Arbovirus infections in humans in New South Wales. Seroepidemiology of the alphavirus group of togaviruses. Med J Aust 1984; 24:700-704. 5. Rich G, Mckechnie J, McPhan I, Richards B. Laboratory diagnosis of Ross River virus infection 1993. Communicable Diseases Intelligence 1993; 17(10): 208-209.

6. Carter IW, Smythe LD, Fraser JR, Stallman ND, Cloonan MJ.

Detection of Ross River virus immunoglobulin $\mathrm{M}$ antibodies by enzymelinked immunosorbent assay using antibody class capture and comparison with other methods. Pathology 1985;17:503-508. 